\title{
La Práctica de la Medicina de Familia en el National Health Service
}

\author{
Candelaria Ayuso Rayaa, Francisco Escobar Rabadán ${ }^{b}$, Clotilde Boix Grasc, \\ Penélope Navarro Piñeirod, Celia Ramos Guisande ${ }^{e}$
}

a Residente de Medicina Familiar y Comunitaria. Unidad Docente de Albacete (España).

${ }^{\mathrm{b}}$ Médico de Familia. Centro de Salud Universitario Zona IV de Albacete. Profesor Asociado de la Facultad de Medicina de Albacete (España).

${ }^{c}$ Médico de Familia. Jefa de Estudios de la Unidad Docente de Atención Familiar y Comunitaria de Albacete (España).

${ }^{d}$ Médico de Familia. Salaried in The Almshouse Surgery. Trinity Medical Centre. Wakefield (United Kingdom).

e Médico de Familia. Partner in The Almshouse Surgery. Trinity Medical Centre. Wakefield (United Kingdom).

Correspondencia: Candelaria Ayuso Raya, Centro de Salud Zona IV, c/ Seminario $\mathrm{n}^{\circ}$ 4, 02006 Albacete (España). Correo electrónico: candeayuso@hotmail.com

Recibido el 29 de noviembre de 2010.

Aceptado para su publicación el 16 de diciembre de 2010.

\section{RESUMEN}

En los meses de abril, julio y agosto de 2010, los autores de este artículo nos marcamos como objetivo conocer de primera mano la atención primaria en el Nacional Health Service (NHS).

Así pues, la experiencia de participación activa de cada uno de nosotros en un modelo referente en atención primaria responde a una fuerte motivación personal, suponiendo un especial atractivo para la configuración de este artículo el poder extrapolar nuestras apreciaciones e impresiones, claramente marcadas por los diferentes momentos de nuestra trayectoria profesional en la valoración de las diferencias estructurales, organizativas, de los lugares de trabajo, del análisis de los equipos, de la formación y de la figura del que hoy por hoy es el principal protagonista de un modelo de salud pionero en Europa.

Palabras clave: Prestación de Atención de Salud, Atención Primaria de Salud, Médico de Familia.

\section{ABSTRACT}

The Practice of Family Medicine within the National Health Service

In the months of April, July and August 2010, the authors of this article set themselves the objective of learning about Primary Care in the National Health Service (NHS) from first hand experience. The active participation of each of us in a reference primary care model stems from a strong personal motivation. Our findings and impressions, clearly influenced by the different periods in our career, were very useful in preparing this article. Through them we could assess the structural and organisational differences of the work places, the team analysis, the training and the figure that today is the main protagonist of a pioneering health model in Europe.

Key words. Delivery of Health Care, Primary Health Care, Family Physician.

\section{LA PRÁCTICA DE LA MEDICINA DE FAMILIA EN EL NATIONAL HEALTH SERVICE}

"Only happy doctors are good doctors" (2010 European WONCA Conference)

En un momento en que nuestro sistema de atención primaria muestra signos de estancamiento, lamentablemente relegado a labores auxiliares de la omnipotente medicina hospitalaria, se hace cada vez más necesario "reformar la reforma".

Para aquellos profesionales que estamos motivados por esta tarea supone un especial atractivo la búsqueda de referentes en otros sistemas de salud afines. Por eso es natural que volvamos la mirada al National Health Service (NHS) británico, referente indiscutible por su trayectoria histórica y por haber sido uno de los modelos principales que inspiró nuestro sistema de atención primaria. Así que no hemos querido dejar pasar la oportunidad de conocer de primera mano este medio asistencial, en una concatenación de circunstancias favorables: rotación externa en un centro de salud inglés de una residente de tercer año de medicina de familia, oportunidad para el tutor de realizar esa misma actividad y estancia de la jefa de estudios de la unidad docente en 
centros de salud de Londres, en un intervalo de pocos meses. La gran coincidencia en los puntos de vista de los tres nos hizo plantearnos la redacción de este artículo, que hemos querido completar con los puntos de vista de dos de las General Practitioners (GP) responsables de supervisar las estancias formativas de los dos primeros.

Hemos optado por una estructuración muy libre del artículo, en donde cada uno pudiera aportar su punto de vista, aunque intentando seguir un esquema que facilite su lectura.

Entendemos que nuestra inquietud no es algo aislado. En este sentido hay que subrayar que el Ministerio de Sanidad y Política Social desde hace unos años está impulsando y coordinando el Marco estratégico de la atención primaria en el siglo XXI (Proyecto AP-21) ${ }^{1,2}$, en el que colaboran Comunidades Autónomas, Sociedades Científicas y Asociaciones de Pacientes. El objetivo es alcanzar una atención primaria de calidad, accesible a todos de manera equitativa, orientada al ciudadano, con una alta capacidad de resolución, que potencie la continuidad asistencial, con organización y gestión eficientes y que garantice la participación y el desarrollo de sus profesionales.

El Pleno del Consejo Interterritorial del Sistema Nacional de Salud (CISNS) del 11 de diciembre de 2006 aprobó una declaración en la que consideraba adecuado el Marco Estratégico para la mejora de la atención primaria. En ella se señalaba que en un horizonte de seis años (2007-2012) las CCAA deberían desarrollar las medidas que, en el ámbito de sus competencias y con la adaptación precisa a la realidad territorial, hicieran posible la consecución de los avances que planteaba este Marco para la mejora de la atención primaria de salud en España.

Las CCAA han elaborado sus propios planes de mejora teniendo en cuenta el Marco Estratégico AP-21, y por parte del Ministerio se han apoyado proyectos dentro del Plan de Calidad que impulsan líneas de actuación contenidas en estas estrategias, poniendo de relieve, para ser tenidos especialmente en consideración, los desarrollos encaminados a incrementar la capacidad de resolución de los servicios, los cuidados sanitarios domiciliarios, la mejora en la coordinación entre atención primaria y atención especializada y la disminución de la carga burocrática de los profesionales.

Así pues, el objetivo planteado en las primeras evaluaciones ha sido el de conocer la situación de la atención primaria, atendiendo fundamentalmente a los aspectos estructurales, de la organización y de la gestión; la percepción de los ciudadanos; la actividad y calidad del proceso de atención y la contribución de la atención primaria a la obtención de resultados de salud. Este informe constituye la necesaria referencia como punto de partida para seguir la evolución de la atención primaria desde la aprobación de las estrategias de AP-21.

Pero la verdadera realidad a la que nos enfrentamos a diario los médicos españoles se aleja notablemente de la consecución de avances, encontrándonos, como datos más significativos, con niveles de infra-dotaciones de los recursos humanos básicos, así como del gasto sanitario imputado a la atención primaria.

Qué radical diferencia con el sistema de salud británico ${ }^{3}$, completamente centrado en la figura del GP.

\section{ESQUEMA ORGANIZATIVO EN LA ATENCIÓN PRIMARIA BRITÁNICA}

Intentaremos resumir la organización básica de la atención primaria británica. Para un conocimiento más profundo recomendamos la lectura del Oxford Handbook of General Practice ${ }^{4}$.

\section{El trabajo del GP}

Su actividad asistencial se organiza por bloques en horario de mañana y/o tarde, siendo frecuente que trabajen sólo 4 días a la semana. En cada bloque la agenda incluye 14 o 15 pacientes, citados cada 10 minutos. Los pacientes pueden pedir cita con cualquiera de los médicos del centro de salud, independientemente de con cuál estén asignados. Si requieren atención urgente llaman al servicio de triage, del que hablaremos más adelante. Por turno rotatorio, cada día uno de los médicos del equipo atiende a los pacientes que acceden a los servicios del centro de salud de esta manera (podríamos llamarles "pacientes que solicitan ser visitados en el día"), que intercala entre huecos de su agenda en la que puede haber también pacientes de la cita previa normal.

Como hemos señalado, el GP representa el principal protagonista en la toma de decisiones con relación a la asistencia a los pacientes, aunque puede contar con el concurso de otros profesionales médicos, a través de interconsultas. En relación con éstas podemos resaltar que el paciente puede elegir 
entre diferentes hospitales que se le ofertan. Otro dato importante, el papel que se lleva el paciente no contiene información clínica.

La parte negativa de esta forma de realizar las interconsultas es la tremenda burocracia, ya que se pasan muchísimo tiempo redactando informes dirigidos personalmente a cada especialista, que se envía por correo (generalmente ordinario). La contestación a la interconsulta la recibe el GP por e-mail o por correo convencional, que tiene que ir revisando y archivar/actuar según corresponda en cada caso.

No es esta la única fuente de burocracia: impresiona ver los montones de papeles que reciben, cartas o las lista de e-mails pendientes de leer, o la emisión de certificados sobre condiciones de higiene y seguridad en las viviendas que puedan comprometer la salud de los convivientes ("housing").

En cuanto a la prescripción, ésta está muy condicionada por los fármacos eficientes (p. ej. prácticamente solo utilizan lansoprazol como IBP). En la hoja de medicación se indica el precio de cada fármaco elegible. Hay muchísimas situaciones en las que los pacientes no pagan nada por la medicación (niños, estudiantes, mayores de 60 años, enfermos crónicos, etc.). Los fármacos que no precisan prescripción (p.ej. paracetamol, o AINEs a dosis bajas, como $200 \mathrm{mg}$ de ibuprofeno) es más barato comprarlos libremente, en un supermercado, que comprarlo con receta.

Los médicos tienden a "especializarse" en alguna tarea. Hay "miniespecialistas" en diabetes, contracepción, cirugía menor (incluye, además de la pequeña cirugía, crioterapia, infiltraciones, colocación o retirada de implantes anticonceptivos, etc.). Es cuestión de hacer un "training", que hay que pagar, aunque el centro de salud o el área de salud suelen correr con los gastos.

Los General Practitioner with especial interest (GpwSI) requieren un entrenamiento que, en función de la especialidad (Cardiología, Oftalmología, Otorrinolaringología, Dermatología, Musculo-eskeletal, etc.), oscila entre 18 y 24 meses, incluyendo la rotación en un Hospital.

Para tutorizar residentes (GP registrar) hay que estar acreditado. El residente tiene su propia consulta.

No suele haber sustituto para el profesional que está de vacaciones, y el trabajo se acumula para los que se quedan, así que hay que hacer más bloques de consulta, pero también se acumula la labor administrativa, y son menos a la hora de repartir avisos, de forma que tocan a más.

\section{Enfermería}

Hay diferentes tipos de enfermeras. En relación con la asistencia a las urgencias hablaremos de las triage nurses. Además están las practice nurses, districts nurses y las community matron. Mención aparte merecen los health visitors, encargados de la asistencia a la infancia.

Las practice nurses son las únicas que forman parte propiamente del equipo, y trabajan en consulta en el centro de salud.

Su trabajo no es muy diferente al que se hace en España en cuanto a la organización de la agenda: pacientes citados cada 15 minutos, y predominio de revisión de crónicos. Hasta ahí las semejanzas, porque además se ocupan de muchas más tareas: contracepción, control de ciertos tratamientos (como el bloqueo hormonal en cáncer de próstata), consejo a viajeros a zonas con riesgos para la salud, vacunas, deshabituación tabáquica, etc.; y dentro de los crónicos una amplia gama de patologías (por supuesto que predominan HTA y diabetes, pero además hay cardiopatía isquémica, abordaje del RCV, obesidad, asma, EPOC, epilepsia, IRC, artrosis, etc.).

Algunas enfermeras son prescriptoras, es decir, pueden firmar recetas. Para ello tienen que haber realizado un "training" de 6 meses. Tienen protocolizado lo que pueden recetar en procesos agudos y pueden firmar las recetas de tratamiento prolongado de los enfermos crónicos que siguen en la consulta.

La nurse practitioner es una enfermera que ha realizado una formación postgrado de tres años y que atiende básicamente consulta a demanda, tiene una agenda similar a la de los médicos, cada $10 \mathrm{mi}-$ nutos, y atiende todo tipo de patología. El paciente puede elegir quien le atiende, el médico o la nurse practitioner. Sorprende la capacidad de tomar decisiones de esta enfermería. Realizan la historia, exploran, realizan el diagnóstico y deciden el tratamiento o la remisión a determinados servicios (fisioterapia, psicoterapia o counsellors, atención a enfermedades de transmisión sexual, anticoncepción, etc.), solo en casos excepcionales remiten al paciente al médico de familia. No todos los centros tienen nurse practitioner. 
Las community matron son enfermeras encargadas de visitar a los pacientes crónicos en su domicilio. Este nivel de asistencia facilita la disminución en la demanda asistencial en las urgencias hospitalarias.

La clave para realizar cualquier actividad asistencial es haber hecho un "training" previo. Ahora bien, esta cualificación también permite tomar decisiones. Así, por ejemplo, los pacientes que van a viajar a zonas de riesgo consultan directamente con la enfermera, y ella decide, administra las vacunas que correspondan e indica la profilaxis antipalúdica si corresponde.

Es decir, a más preparación más trabajo, pero también mayor remuneración.

Las district nurses dependen de la comunidad y su trabajo es la asistencia a domicilio: curas, control de pacientes crónicos con dificultad para desplazarse al centro de salud, administración de medicación a estos pacientes, etc.

En cuanto a los health visitors, se trata de nurses con formación específica en la asistencia a niños. Realizan una visita puerperal y llevan el control del "niño sano" en el centro de salud. Acuden también a domicilio ante la sospecha de cualquier problema en el ámbito familiar que pueda afectar a los niños.

Tanto district nurses, community matrons como health visitors, aunque no forman parte del equipo, utilizan la infraestructura del centro de salud.

\section{Otros miembros del equipo}

Receptionist: Son el elemento organizativo básico de los centros de salud. Se encargan de las agendas, dar las citas, etc.

Practice manager: es una especie de director de gestión del centro de salud.

Administrativos y secretarias: pueden realizar tareas de apoyo a los diferentes miembros del equipo.

Health Assistant: personal sin titulación específica, pero que ha hecho un training en una cierta tarea, como por ejemplo extracciones (phlebotomist), electrocardiogramas o espirometrías. A veces se trata de recepcionistas a los que se ha facilitado esa formación por interés del propio equipo.
En los centros de salud trabajan otros profesionales de apoyo que no son miembros del equipo de atención primaria. Entre ellos podemos destacar: midwife, dietician, musculo-eskeletal, podiatrist, counsellors, etc.

Aquí no hay trabajadores sociales en los equipos, y la relación con ellos es puntual.

\section{Urgencias}

En muchos centros de salud se han establecido servicios de triage. Todos los pacientes que quieren ser vistos en el día llaman por teléfono y una recepcionista clasifica su problema. Salvo que ésta dirija de entrada al paciente hacia un servicio específico de urgencias, una enfermera se pone en contacto con ellos y decide qué se hace: puede resolver el problema por teléfono, puede citarle para verle en el centro de salud, puede darle una cita con el médico de "visita en el día", o puede indicarle también que acuda a otro sitio:

- De 8,00 a.m. a 8,00 p.m. funciona el Walk-in centre, que es como un servicio de urgencias al que los pacientes acuden sin necesidad de cita.

- A partir de 6,00 p.m. funciona el Out of hours. El servicio de triage termina a las 4,00 p.m. Entre las 4,00 y las 6,00 (cuando se cierra el centro de salud) el médico atiende directamente las demandas.

Las triage nurses tienen recetas firmadas por los médicos para tratar ciertos problemas:

- ITU no complicadas: trimetoprim (cefalexina en caso de alergia).

- Impétigo: flucoxacilina (eritromicina en alérgicos).

- Vaginitis: clotrimazol.

- Conjuntivitis (en mayores de un año): cloranfenicol.

- Dolores/fiebre: paracetamol o ibuprofeno.

Así resuelven muchos problemas menores, pero si la situación del paciente se sale de lo que marca el protocolo la enfermera lo consulta al médico, que puede decidir la prescripción sin ver al paciente, o bien optar por visitarlo personalmente.

\section{Reuniones de equipo}

No recuerdan en nada a las nuestras: allí nadie grita, es más, el tono puede ser monótono, incluso aburrido.

Los laboratorios también organizan alguna actividad, pero tienen mucha menos presencia que en 
España. La visita médica la organizan una vez a la semana, con todos los médicos juntos. El laboratorio correspondiente organiza un lunch (unos sandwiches y unas bebidas -no alcohólicas-) en el mismo centro. Dejan unos 10 minutos al laboratorio y a continuación hacen sesión clínica o reunión de equipo.

Cuando se celebra un "meeting", en el que participan todos los profesionales del equipo, el centro de salud se cierra a las 12 de la mañana. A las 12,30 "lunch" (generalmente un catering sencillo) y a las 13 horas a la sala de reuniones. Trabajo en grupos, discusión conjunta, etc., nada nuevo con relación a la dinámica del grupo o las conclusiones, pero la estrategia organizativa es perfecta. A partir de las 12 los pacientes son atendidos en los servicios correspondientes para urgencias y todos los profesionales acuden a una actividad formativa en su horario de trabajo, que además se ve como un momento lúdico para compartir comida y conversación con los compañeros.

Se celebran también los llamados TARGETs (Time for Audit, Research, Guidelines, Education and Training), que son reuniones mensuales con todos los GP del distrito (Target In) y los Target Events, o reuniones bimensuales con profesionales de atención primaria (médicos, enfermeras, otros profesionales relacionados con la reunión) y hospitalaria, donde se tratan temas organizativos y se imparten sesiones clínicas.

\section{La formación continuada}

El tema de la formación es muy importante para ellos. Están muy en boga los trainings que ya hemos comentado. Si quieren poder hacer actividades extra, que generalmente están muy bien pagadas, tienen que hacer la formación específica.

Por otra parte, los médicos tienen que acreditar anualmente su "appraisal", y obtener cada 5 años la acreditación del Royal College of General Practitioners (RCGP: el colegio de los GP). El appraisal anual requiere mucho tiempo por parte de los médicos, e incluye análisis de las reclamaciones, audits, encuesta de satisfacción de los usuarios, SEA (significal event), y los PUNs and DENs. Los SEA hacen referencia a determinadas situaciones que pudieran haber resultado incorrectas o susceptibles de ser mejoradas, y se exponen en un meeting con otros compañeros. Los PUNs and DENs se refieren a situaciones especiales que analizan las necesites particulares de los pacientes y el nivel de satisfacción de sus expectativas.
Para los residentes también hay evaluaciones fuertes, y la opción de acreditar su competencia para trabajar como GP (es opcional pero prácticamente indispensable para que les contraten).

\section{Remuneración}

Como se ha comentado, trabajan por bloques, que es una consulta de unas 14 citas de 10 minutos; puede ser de mañana y/o tarde; y se puede elegir el número de ellas a hacer semanalmente, aunque lo común es que sean 7-8. Cada bloque supone unas 8000-9000 libras anuales.

Cada centro de salud se organiza de forma completamente autónoma, por lo que es difícil extrapolar a un patrón general. Hay centros donde un solo GP asume toda la carga de trabajo, y se apoya en la contratación de algún médico y personal auxiliar. Trabaja como un esclavo mañana y tarde y además hace guardias los fines de semana. Pero lo más habitual es que un equipo de médicos (partners) organice una especie de cooperativa. Estos pueden ganar 120.000 pounds brutas anuales.

Las relaciones con la gerencia prácticamente las lleva el director de gestión (practice manager), aunque las decisiones son de los partners. Estas relaciones prácticamente se centran en la "cartera de servicios" que oferta el centro de salud. Que asumes llevar la contracepción, pues tanto, que llevas la cirugía menor, tanto, que llevas la insulinización de los diabéticos, tanto, que llevas el tratamiento de supresión hormonal en el cáncer de próstata, tanto, etc.

El sistema de incentivos es el llamado QOF (The Quality and Outcomes Framework) ${ }^{5}$ : los centros de salud reciben una cantidad de dinero en función de los resultados conseguidos. Desde que fueron introducidos en 2004 han sufrido varias revisiones. En la de 2008/2009 incluían cuatro componentes o dominios, cada uno de los cuales consta de un conjunto de indicadores:

- Atención clínica: 80 indicadores en 19 áreas clínicas (cardiopatía isquémica, insuficiencia cardíaca, HTA, etc.).

- Organización: 36 indicadores en 5 áreas (registros e información, información para los pacientes, educación, gestión del centro y de farmacia).

- Experiencia de los pacientes: 5 indicadores que se relacionan con la duración de las consultas e informes de los pacientes. 
- Servicios adicionales: 8 indicadores en 4 áreas de servicio que incluyen screening de cáncer de cérvix, seguimiento del niño sano, y servicios relacionados con la maternidad y la contracepción.

Ahora este sistema está en proceso de cambio, debido fundamentalmente a la crisis económica y al cambio de gobierno.

\section{Los pacientes}

Ya se ha comentado lo educados que se muestran en la consulta y lo considerados que son con el médico (el tema de la consideración del médico merecería un capítulo especial: todo el mundo se dirige al médico como " $\mathrm{Dr}$ ", incluyendo receptionist, nurses, etc.). Por lo demás, muchas similitudes con los nuestros. Quizás allí son un poco histriónicos, debe ir en la forma de ser británica, y le echan mucha "teatralidad" a las explicaciones que dan, tanto los pacientes como los acompañantes. Claro que tienen mucho tiempo para explayarse. Incluso se les alienta: no hace falta prevenir el "by the way", sino que continuamente se les pregunta si se puede hacer algo más por ellos. Se recurre mucho a darles información escrita, generalmente obtenida de páginas web (utilizan mucho esta web: http://www.patient.co.uk/ $)^{6}$.

Para un médico español es muy llamativo lo que ocurre con relación a las bajas (Sick Note): te piden 4 semanas de baja como la cosa más natural del mundo. En un impreso tamaño cuartilla escribes el nombre del paciente, el diagnóstico y la duración (pueden ser semanas o meses). Los primeros 7 días los pacientes no necesitan presentar la baja a la empresa. Ocasionalmente el paciente requiere la emisión de un Self Certificate para su trabajo. En caso de solicitar un informe de acreditación de su enfermedad en este periodo (Private Sick Note), el Centro le cobra una cantidad (6-10 Libras), ya que no es algo obligatorio. Desde luego menos burocracia, porque no hay que hacer partes de confirmación.

Y no solo las bajas, también es muy sorprendente la cantidad de personas que viven de lo que llaman "benefits". Pacientes que pueden llevar años cobrándolos por "enfermedad" van a por el informe del médico para seguir viviendo del estado.

\section{EL PUNTO DE VISTA DE LA RESIDENTE}

Antes de partir mi tutor me aconsejó que mis impresiones las fuera relatando a modo de blog, por lo que contaré los detalles más relevantes.
Como primer impacto, para un residente español, acudir a la casa del residente inglés es la invitación a la mansión donde practican el camino de la excelencia. Para ambos se torna largo y exigente, pero ellos juegan con ventaja, su excelencia educacional va dirigida por un camino empedrado de conceptos fundamentales como la sostenibilidad del sistema, los valores compartidos, la rendición de cuentas y por supuesto los resultados equilibrados y el valor para los clientes, que en definitiva constituyen los principales atributos de la ética en las organizaciones ${ }^{7}$.

Maduran en experiencia y habilidades en su entrevista clínica durante la residencia. Practican la adquisición y la mejora de conocimientos a través de un duro role-playing como herramienta de distinción ante el paciente, constituyendo una de las "evaluaciones fuertes" que deberán aprobar para finalizar su periodo formativo.

Disponen de la referida "Casa del Médico", que es la casa de los residentes y sus tutores. Habitualmente es un espacio habilitado en un Centro de Salud, donde comparten espacios destinados para reuniones, biblioteca y consultas virtuales.

Mi contacto con un grupo de residentes ingleses tuvo lugar una semana antes de partir a España. Invitada por el General Practitioner registrar (GP registrar) de mi centro de salud, acudí a una de sus reuniones. Reconozco que me sentí perpleja y al mismo tiempo admirada por su formación.

Una de las habilidades en las que más trabajan y más esfuerzos destinan es en la capacidad de comunicarse, no de forma correcta, diría que de forma exquisita. Trabajan en educación y capacidad de comunicación con el paciente, capacidad para compartir los conocimientos médicos, de establecer silencios "programados" y hábiles para la toma de decisiones.

Trabajan en su propia consulta (informan a los pacientes que van a ser vistos por un médico en formación), se sientan junto a ellos, comparten su historia clínica, la pantalla de su ordenador, a pesar de establecer como única barrera el contacto personal.

No llevan equipos de trabajo (bata, zuecos), no son paternalistas y a veces impresiona la naturalidad con la que toman un té a lo largo de la entrevista clínica. Son conocedores del respeto implícito de sus compañeros de profesión y de sus pacientes, se sienten el primer escalón del sistema, por lo que irradian un aura especial, permitiéndose el training 
por su "especial interés en determinadas patologías", por lo que el sistema les facilita la subespecialización, lo que los refuerza como Médicos, en su centro de trabajo y en su nómina anual.

¡Cómo impacta a los sentidos un día de trabajo en Inglaterra! Suelos enmoquetados, grandes sillones junto a la mesa del médico, tensiómetros, oftalmoscopios y otoscopios situados junto a la silla del paciente, camillas con cortina, etc., y todo bajo lo que he denominado "la armonía del silencio", la cortesía, la empatía del médico con el paciente y viceversa, la escucha activa, los formalismos con los que los pacientes explican su sintomatología y el respeto implícito en esta relación puntual.

Por un momento, y sigo impactada por la moqueta, me pregunto si el vómito, las secreciones o los fluidos corporales son cosas de educación.

Creatividad e innovación es lo que nos exigen a los residentes españoles ante un modelo poco flexible y condicionado en la consecución de avances, en el que la calidad en el proceso de atención asistencial y la contribución de la atención primaria a la obtención de resultados de salud está condicionada por una organización sanitaria pública impregnada de verticalidad burocrática.

En el "ejercicio diario" de nuestras tareas, nuestro modelo se debate entre el individualismo motivacional, la práctica de la excelencia clínica y las dificultades en la gestión de recursos sanitarios.

Es sorprendente cómo se gestiona la información. Existen folletos, boletines, dípticos, carteles informativos en las salas de espera, y una web específica con información destinadas a pacientes y facilitadas en la consulta a través del médico.

Y qué decir de las reuniones de equipo semanales (practice meeting) dirigidas por la practice manager, con el fin de diseñar y mantener un entorno en el que los individuos, trabajando juntos de manera eficiente, logren determinados objetivos, esenciales para la adecuada toma de decisiones conjuntas.

A través de la participación conjunta con los partners y bajo unas directrices muy específicas con gran capacidad de liderazgo, sus esfuerzos van dirigidos a la planificación, coordinación y control del rendimiento global, la revisión de los estándares de atención, la cartera de servicios, así como la situación financiera del centro de salud.
La información, por tanto, va dirigida a todo el equipo, facilitándoles ayuda en técnicas de comunicación.

Continuando con las reflexiones individuales, el enfoque estructural sobre el organigrama inglés choca frontalmente con nuestro sistema público financiado con impuestos. Si lo gestionamos por las reglas de la Ley Pública, si tenemos trabajadores que han opositado y sus retribuciones son por categoría profesional, ¿cómo luchamos frente a la gestión privada Inglesa?

La pirámide de responsabilidades depende de un sistema vertical que debería ser horizontal. ¿Cómo vamos a imitar una cultura de reconocimiento, de comunicación equilibrada, de respeto implícito, si hemos traspasado nuestros propios límites?

Ahora, tras unos meses en la consulta, añoro la eficiencia del sistema, el silencio, el respeto, la pluripotencialidad de un equipo multidisciplinar de profesionales sanitarios y administrativos trabajando de forma conjunta.

Y aunque sienta que no estoy en el vértice de la pirámide de mi SNS, siento como residente el valor clínico adquirido en el modelo español. Practicar en una consulta virtual con un modelo ya creado y valorado desde 1948 es apostar por el futuro.

El sistema de aprendizaje integral, la implicación en aspectos teóricos, clínicos, la accesibilidad en metodología de la investigación, conocer a los pacientes del cupo, su entorno familiar, conocer su historia clínica equilibra las sensaciones y los sentimientos de añoranza añadiendo valor a mi experiencia como médico en formación.

Como residente, nuestros tutores españoles, con su ejercicio profesional, nos inquietan por el futuro, que es nuestro futuro, $y$, por tanto, el principal impulsor de cambios. Habrá que seguir trabajando para lograr resultados personales equilibrados, soñar con construir nuestra "casa del médico español" y no decaer en las aspiraciones individuales en la lucha por la excelencia, la productividad, la eficiencia, que al fin y al cabo, tal y como nos demuestran con su trabajo diario nuestros compañeros ingleses, es cosa de todos los profesionales sanitarios.

\section{EL PUNTO DE VISTA DEL TUTOR}

Mis primeros días fueron una sucesión de impactos: 
1. Las consultas están separadas de la sala de espera por un pasillo. Es increíble no oír el ruido de fondo. Pero esto no es todo, jnadie interrumpe la consulta! No llaman a la puerta, nadie irrumpe por las bravas, no suena el teléfono. Los pacientes son llamados a consulta a través de una pantalla en la que aparece su nombre cuando el médico lo marca en la hoja de visita del día. Cuando el paciente llega al centro de salud activa su nombre en una pantalla (si no se maneja con este sistema simplemente da su nombre a las recepcionistas).

2. Pocos pacientes y mucho tiempo para dedicarles. A pesar de esto puede haber retraso, pero no provoca ningún comentario de los pacientes. En nada cambia su actitud correcta, de agradecimiento y de reconocimiento con el trabajo del médico.

Posteriormente, el conocimiento del trabajo de diferentes profesionales, la participación en sesiones y reuniones de equipo, y, en definitiva, el día a día de la actividad asistencial, fueron configurando puntos de vista más estructurados.

El GP es mucho más que el gate keeper (es decir el que regula la entrada al sistema), es el verdadero centro de la toma de decisiones con relación a la asistencia a los pacientes. En este sentido es muy llamativa la forma en que otros especialistas se dirigen a él, la deferencia con que contestan las interconsultas.

Para comprender el marco de trabajo que presencié pueden ser útiles algunos datos:

- Alrededor de 12.000 pacientes registrados en el centro de salud (un centro principal: Almshouse Surgery, y una especie de consultorio: Sandal Castle, pero que no es un simple "consultorio local" en el sentido nuestro, sino un centro de salud en toda regla).

- Ocho médicos (7 partners, o sea "propietarios del negocio", y una salaried), más un GP registrar (residente). Si bien hay que tener en cuenta que no todos trabajan a tiempo completo, es bastante llamativa la ratio pacientes/médicos.

- Enfermería: 4 district nurses, 4 practice nurses, más el equipo de triage nurses (que es compartido con un centro de salud vecino).

Con relación a los pacientes, para la mentalidad española sorprende mucho aquella sociedad. Hay 2 países: están los que tienen un trabajo en condiciones, viven en zonas residenciales, tienen buenos coches, sus hijos estudian en magníficas universi- dades, etc., y están los que viven en bloques de viviendas sociales, con un trabajo mal remunerado, o directamente malviviendo de la "caridad pública". Son tremendamente comunes (al menos en los pacientes que consultan en el centro de salud) historias de vida complicadísimas, que generalmente empiezan por embarazos en edades tempranas (probablemente precedidos también por historias de maltrato en la infancia). Curiosamente un país con unos servicios de asistencia contraceptiva excepcionales (todos los métodos anticonceptivos son gratuitos y fácilmente accesibles) tiene la tasa más alta de embarazos en adolescentes del mundo occidental. Entre otras razones, hay que considerar los "benefits" que reciben por tener hijos, aparte del dinero que el estado ingresa para cada niño y del que puede disponer al llegar a la mayoría de edad. Podemos sumarle la cantidad de alcohol que consumen, lo mal que comen (a base de comida rápida/basura, en la gama alta de lo hipercalórico), que acumulan rápidamente unas obesidades en el rango mórbido, y que muchos alcanzan la diabetes tipo 2 con frecuencia en la treintena. Así no es de extrañar que desde bien jóvenes estén descontentos con la vida, tomen antidepresivos, etc.

Desde luego que la ecuación pocos pacientes con mucho tiempo para dedicarles es fácil de resolver. De todas formas lo que más me ha impactado de la experiencia inglesa no tiene tanto que ver con la cantidad sino con la calidad. He descubierto lo que podríamos Ilamar "la dignidad de la profesión". Desde la residencia nos acostumbramos a "ir a la guerra", te "echan a las fieras" y si sobrevives te curtes (muchos se queman ya desde esa etapa, claro). No solo no conocemos la dignidad de la profesión (al menos para mi ha sido un absoluto descubrimiento) sino que incluso se "nos vacuna" contra estas aspiraciones: el sistema nos acosa con su política de "el cliente siempre tiene razón" (iqué diferente del NHS, donde si el paciente se comporta incorrectamente o incumple citas se le echa sin más del centro de salud!). Algunos incluso (y aquí tengo que entonar un "mea culpa") nos hemos dejado impregnar de una cierta mojigatería, confundiendo los derechos ciudadanos con un "todo vale", y aceptando de buen grado sueldos que no han estado acordes con el nivel de desarrollo económico del país.

Quizás ha sido la concatenación de acontecimientos lo que me ha llevado a "la revelación". Podría estar satisfecho con el sueldo, pero si te lo bajan, y a renglón seguido te trasladas a un medio laboral radicalmente distinto... Entiendo perfectamente por qué magníficos médicos de familia españoles están 
trabajando en otros países y no quieren volver. Lo que no es entendible es que a nuestras autoridades sanitarias no les preocupe haber desperdiciado este inmenso tesoro intelectual, y los hayan sustituido por portadores de títulos de dudoso origen (sin formación de postgrado en cualquier caso).

El balance más sencillo en cuanto a conclusiones puede plantearse como qué me gusta y qué no me gusta de cada servicio de salud.

\section{NHS}

Me gusta:

- El pasillo que separa la sala de espera de la consultas.

- La calidad del trabajo del médico, en cuanto a disponibilidad de tiempo, ausencia de interrupciones, etc.

- Consideración del médico, tanto en el entorno profesional como por parte de los pacientes.

- La posibilidad de organizar equipos con profesionales afines.

- La gestión de los centros.

- El sueldo.

No me gusta:

- Mucha burocracia.

- Muchos domicilios.

- Demasiado "protocolo" (no en el sentido de "protocolizado", sino de "protocolario").

- Mucha dispersión en la asistencia (demasiados profesionales pueden verse implicados).

- La tendencia de los GP a hacerse "miniespecialistas".

\section{SESCAM}

Me gusta:

- Tener mi cupo de pacientes: les conozco personalmente a la mayoría y eso es una fuente de información de valor incalculable.

- Compartir su asistencia con enfermera y residentes.

- La accesibilidad de los pacientes.

- Lo variado del trabajo.

No me gusta:

- Que no haya un número límite en el cupo de pacientes (todo lo que sea sobrepasar los 1.500 es excesivo).

- Los excesos que han devenido de la buena accesibilidad de los pacientes.

- La poca consideración por parte del servicio de salud, de otros especialistas médicos, incluso de muchos pacientes.

- Ser los profesionales médicos peor pagados del mundo occidental.

Hay dos aspectos organizativos sobre los que tenía un especial interés. Por una parte el hecho de que los pacientes puedan pedir cita con cualquier médico del centro y no solo con el que están adscritos. Mi impresión, desde luego condicionada por mis preferencias por la asignación personal, es que la atención del paciente puede ser errática. $Y$ esto en un equipo en que hay coincidencias en cuanto a la forma de trabajo, y se acepta la protocolización de muchas actividades; qué sería en equipos donde los profesionales han ido cayendo en función de las circunstancias administrativas del momento (oposiciones, traslados, comisiones, listas de interinidades, etc.). Lo vemos de hecho en las consultas hospitalarias: qué fácil es caer en "echar balones fuera" y en "marear la perdiz".

Otro aspecto en el que tenía mucho interés era en el trabajo de enfermería, especialmente con relación al "triage". Me ha gustado la responsabilidad que asume la enfermería en la toma de decisiones, sin embargo veo excesivamente parcelado el trabajo. Mi impresión con respecto al "triage" ha estado muy condicionada por el hecho de ser época de vacaciones y haber poca demanda. Sin embargo, más allá de las circunstancias puntuales, creo que hay una lectura clara de lo aprovechable de esta tarea. Aunque en la práctica muchos ya lo hacemos, creando huecos de cita para "urgencias" (deberíamos hablar más bien de "huecos para pacientes sin cita previa"), sería importante diferenciar agendas de "demanda demorable" (para la que se debería admitir la existencia de "lista de espera") y "demanda no demorable" (visita en el día).

¿Quién decide si es o no demorable?

No debería ser el paciente, pero tampoco el auxiliar administrativo. En este sentido sí que veo útil que profesionales de enfermería, con formación específica, realicen esta tarea. Ahora bien, yo propondría un sistema más ágil que el británico. Una enfermera de guardia recibe esas llamadas en su misma consulta, y les da respuesta automática: ser valorado por ella misma en consulta, cita en la agenda de "visita en el día" del médico, o cita en la "agenda de demanda demorable". Incluso podría tomar decisiones sobre adecuación de visita a domicilio, o pasar a otros servicios asistenciales como 112, SUAP o derivación a urgencias hospitalarias. 


\section{EL PUNTO DE VISTA DE LA JEFA DE ESTUDIOS DE LA UNIDAD DOCENTE}

Durante mi estancia en Londres estuve en 2 Centros de Salud, uno en el centro de la ciudad, en el distrito de Westminster, Marylebone Health Center, y otro en el distrito de Lewisham, en la periferia, Rushey Green Practice. Mi sorpresa inicial fueron los edificios en que se encontraban los Centros de Salud. Son locales alquilados por los partners y, dado que los alquileres en Londres son muy elevados, Marylebone Health Center estaba en un sótano, con lo cual había varias consultas sin ningún tipo de ventilación ni luz natural. El Rushey Green Practice estaba en la planta baja de un edificio perteneciente al PCT (Gerencia de AP del área). Nada que ver con el espacio y las condiciones de la mayoría de nuestros centros de salud (son locales que podían servir para cualquier negocio).

La distribución de la consulta también cambia. Nunca hay mesa entre el profesional sanitario y el paciente, se sientan uno enfrente del otro. El paciente puede ver en todo momento la pantalla del ordenador con lo que está escrito o escribe el médico en su historia. El médico nunca lleva bata blanca y viste con corrección, nunca vaqueros o zapatillas de deporte.

En cuanto a la organización del trabajo, ya comentada en apartados anteriores, sorprende el respeto al tiempo de consulta de los profesionales, mientras se atiende pacientes no hay ni una interrupción, ni presencial ni telefónica. Si algún compañero o un residente quieren hacer una consulta, espera en la puerta hasta que sale el paciente, nunca se interrumpe la consulta.

El ritmo de trabajo es muy adecuado, pues están bien distribuidos los tiempos para que la consulta no sea agotadora.

Aunque los pacientes que quieren ser vistos en el día no pueden elegir el médico y esto dificulta la continuidad, registran muy bien toda la información, y el paciente, en general, es consciente de que el profesional que le atiende dispone de toda la información.

Sorprenden también los diferentes tipos de enfermería y las diferentes funciones que asumen según su formación. Los partners contratan enfermería con distintos niveles formativos según las necesidades del centro.
Los Servicios Comunitarios (Community Services), dependientes del área, no del centro, tienen una gran importancia y suponen una gran inversión. Tienen una gran tradición y la población está muy satisfecha con este servicio (district nurse, health visitor, community nurse, time bank, etc.), está muy implementado, pero es muy complejo.

En cuanto a los residentes, la formación es troncal, los dos primeros años comunes para especialidades médicas (Foundation1 y Foundation2), con rotación obligatoria en Atención Primaria. Los residentes pasan consulta solos desde el principio, aunque con menos número de pacientes y con más tiempo (empiezan por 5 pacientes, con un intervalo de 20-30 minutos, y van aumentando progresivamente), no interrumpen al tutor, pero sí comentan todos los días los pacientes que han visto y en muchas ocasiones preparan la consulta con el tutor, que les orienta antes de empezar.

Los que deciden ser médicos de familia (GP), una vez finalizado F1 y F2, tienen 3 años de formación, Registrar 1, 2 y 3, dependen organizativamente de una Unidad Docente (Deanary) ${ }^{8}$, realizan 18 meses en el hospital y 18 meses en el centro de salud. Mientras están en el centro no realizan guardias en el hospital. Pasan consultas 8 sesiones semanales. Cada sesión son 3-4 horas de consulta y participan en todas las actividades del Centro. Hacen guardias hasta las 20 horas, que permanece el centro abierto. Mientras están en el Centro firman un contrato con los partners que reciben del NHS la cantidad necesaria para pagar su salario, que es entre 45.000 y 50.000 libras, según el año de formación. Realizan una actividad similar a la de los médicos del centro. Cuando están en el hospital firman el contrato con éste.

Para iniciar el periodo de Registrar deben estar inscritos en el RCGP y realizar el e-portfolio que establece el Colegio. Otro de los requisitos es tener carnet de conducir y posibilidad de desplazarse de forma independiente, y tener un nivel de inglés oral y escrito demostrado con un examen.

También es el RCGP el que define y evalúa el marco competencial del médico de familia que abarca 12 objetivos en las siguientes áreas: clínica, comunicación con el usuario y el equipo, ética, formación, atención a la comunidad y características del trabajo en AP (complejidad, incertidumbre, prevención y promoción de la salud).

Cada una de estas 12 áreas se puede evaluar, según sus características, mediante uno o varios de 
estos criterios: opinión de los colegas, cuestionario de satisfacción del usuario, observación de la práctica clínica (vídeo), análisis de casos o incidentes críticos, observación estructurada de la práctica clínica o prueba de competencia e informe del tutor de cada rotación, que incluye conocimientos, habilidades y competencia profesional ${ }^{9}$.

Estas evaluaciones se realizan durante el periodo de residencia y al final, y las calificaciones pueden ser: insuficiente, necesita mejorar, competente y excelente. No todos los residentes superan las evaluaciones, pero esto garantiza un nivel de calidad.

Los residentes también tienen oferta de cursos específicos en el RCGP, pero pueden decidir asistir o no en función de sus necesidades de formación o del consejo del tutor. A estos cursos también se puede asistir de forma virtual a través de la web.

Es realmente el RCGP quien define las competencias, el portfolio y realiza el control de las evaluaciones. La Unidad Docente se limita a temas organizativos.

Si un residente no satisface al equipo, por ejemplo porque llega tarde, algo que no toleran, lo "expulsan" del centro, le rescinden el contrato y lo envían a la Unidad Docente para que valore las posibilidades de seguir con su formación.

Creo que los residentes ingleses asumen más responsabilidades durante los primeros años de formación, aunque en los años posteriores sería similar a la que asumen los residentes españoles. Se les exige más autonomía y autoresponsabilización de su formación. El sistema no es en absoluto paternalista con los residentes. Si un residente no se ha preocupado de la calidad de su trabajo y de su formación se le evalúa negativamente. Se les trata como profesionales.

La evaluación final pretende "certificar" la calidad de la formación y esto es positivo para el sistema sanitario.

En cuanto a los tutores, para ser tutor es imprescindible haber realizado un curso de 1 año de duración, por lo tanto el acceso es difícil. En algunos centros solo se acredita un médico como tutor, para garantizar tener al menos un residente anual, aunque todos participan en su formación y supervisión.

En general me ha gustado su organización, aunque algunas cosas son excesivamente complejas y re- quieren grandes infraestructuras difíciles de implementar en nuestro país.

Durante mi estancia he sido consciente de que el NHS ha sido y es nuestro modelo en muchos aspectos, aunque nuestra sociedad es muy distinta y algunas cosas nunca serán comparables. También hay que tener en cuenta que el NHS tiene 60 años y nuestro sistema de salud es mucho más joven.

\section{EL PUNTO DE VISTA DE LA PARTNER}

Algo que me llamó mucho la atención al llegar a Inglaterra fue lo arraigada que está la figura del médico de familia en la cultura y sociedad inglesa. El GP tiene una voz pública y la sabe utilizar con asertividad y confianza, es un profesional al que con frecuencia se le pide opinión sobre asuntos actuales y no es raro verle en las noticias de la BBC discutiendo sobre una nueva ley de gobierno $\mathrm{u}$ otros asuntos que afectan a profesionales sanitarios o a pacientes. También se le consulta abiertamente sobre el manejo del dinero público o aparece en grupos de presión para reivindicar o defender distintas causas.

Llevo 7 años trabajando como médico de familia en el Reino Unido y la experiencia ha sido y está siendo muy positiva. Llegué a este país en un momento en el que los Primary Care Trusts (PCT), que son los equivalentes a las Áreas de Salud en España, necesitaban cubrir plazas de médicos de familia. Al parecer uno de los mayores motivos era que una generación de médicos que llegaron de las antiguas colonias en los años 50 del siglo XX, principalmente de India y Pakistán, entraban en la edad de jubilación y no había suficientes residentes de medicina de familia para cubrir las plazas que iban quedando vacantes. Vine a través de un programa de la embajada británica y estuve algo más de 2 años trabajando de médico asalariado, primero contratada por el PCT y al año por el mismo centro de salud donde había estado trabajando el año anterior. A los dos años y medio, los socios me ofrecieron una "partnership" y decidí asociarme con ellos.

Desde que me hice socia mi rol como médico no ha cambiado en absoluto ya que sigo atendiendo al mismo número de pacientes, pero sí que tengo que involucrarme en temas organizativos y de toma de decisiones, lo que claramente me requiere más tiempo. Pero las ventajas son evidentes, soy partícipe en la toma de decisiones que repercuten directamente en mi trabajo, como por ejemplo el empleo 
de personal sanitario, organización de las citas y consultas, negociación de contratos con el PCT de la provisión de nuevos servicios en el centro de salud, etc. Además cada socio nos hacemos responsables de distintas áreas clínicas u organizativas de los Quality and Outcomes Framework (QOF), lo que conlleva hacer auditorías internas, atender reuniones con el PCT y cursos. Los médicos socios nos reunimos semanalmente después de la reunión de equipo para discutir temas de gestión del Centro de Salud con la Practice Manager, que es la persona que se encarga de la logística. Trimestralmente nos reunimos con el contable para discutir las finanzas.

Estos años trabajados como médico de familia en el Reino Unido han sido muy gratificantes, comenzando por el trato que he recibido desde el principio por mis compañeros sanitarios, de respeto y confianza, que me ha alentado a ser mejor profesional y a saber lo que es trabajar en equipo por el bien del paciente. En mi opinión, la libertad de decisión en la gestión y organización de la prestación de servicios fomenta la creatividad y la búsqueda de eficiencia a la hora de gestionar los recursos y promueve la satisfacción del profesional médico. La capacidad de negociar con el PCT "los puntos sobre las íes" de los contratos fomenta la co-responsabilidad y la involucración del profesional en la aplicación de nuevas directrices gubernamentales.

Me surgen ciertas preguntas, o como dirían los ingleses "some food for thought":

En el ambiente de crisis ideológica y económica actual ¿estamos dispuestos a dejar de lado diferencias políticas para buscar nuevas vías y adaptarnos a los cambios del tiempo?

¿Están nuestros gobernantes preparados y dispuestos para confiar en unos profesionales médicos reconocidos internacionalmente por su buen hacer?

$Y$ finalmente, ¿estamos los médicos españoles preparados para que se nos trate como verdaderos profesionales con la co-responsabilidad que eso conlleva?

\section{BIBLIOGRAFIA}

1. Ministerio de Sanidad y Consumo. Marco Estratégico para la mejora de la Atención Primaria en España: 2007-2012. Proyecto AP-21. Disponible en: http://www.msc.es/profesionales/proyectosActividades/docs/AP21MarcoEstrategico2007_2012.pdf (con acceso el 15-9-2010).

2. Ministerio de Sanidad y Política Social. Marco estratégico para la mejora de la atención primaria en el siglo XXI. Disponible en: http://www.elmedicointeractivo.com/docs/ documentos/INFORMEAP22010.pdf (con acceso el 15-92010).

3. Ayerbe García-Monzón L, Milazzo Loma-Osorio J, PérezPiñar López M, González Martínez S, González López E. Atención primaria en España e Inglaterra; aprender de las diferencias. Opinión de médicos de familia españoles con experiencia en ambos países. Aten Primaria. 2010; 42:10911.

4. Simon Ch, Everitt H, van Dorp F, Schroeder K. Oxford Handbook of General Practice (third edition). Oxford: Oxford University Press; 2010.

5. NHS. Quality and Outcomes Framework. Disponible en: http://www.qof.ic.nhs.uk/ (con acceso el 15-9-2010).

6. Patient UK. Comprehensive health information as provided by GPs and nurses to patients during consultations. Disponible en: http://www.patient.co.uk/ (con acceso el 15-92010).

7. Arcelay A. EFQM: veinte años alumbrando el camino de la excelencia. El modelo se remodela. Rev Calidad Asistencial. 2010; 25:117-9.

8. London Deanery. Disponible en: http://www.londondeanery. ac.uk/general-practice (con acceso el 26-11-2010).

9. Royal College of General practitioners. Disponible en: http://www.rcgp-curriculum.org.uk/nmrcgp/wpba/competence_framework.aspx (con acceso el 26-11-2010). 